\title{
Optical control of photon tunneling through an array of nanometer scale cylindrical channels
}

\author{
I. I. Smolyaninov, A. V. Zayats ${ }^{(1)}$, A. Stanishevsky, and C. C. Davis \\ Electrical and Computer Engineering Department, University of Maryland, College Park, MD 20742 \\ (1) Department of Pure and Applied Physics, Queen's University of Belfast, Belfast BTr 1NN,UK
}

(October 23, 2018)

\begin{abstract}
We report first observation of photon tunneling gated by light at a different wavelength in an artificially created array of nanometer scale cylindrical channels in a thick gold film. Polarization properties of gated light provide strong proof of the enhanced nonlinear optical mixing in nanometric channels involved in the process. This suggests the possibility of building a new class of "gated" photon tunneling devices for massive parallel all-optical signal and image processing.
\end{abstract}

PACS no.: 78.67.-n, 42.50.-p, 42.65.-k

In our recent work [1,2] some of the unusual properties of naturally occurring extremely small pinholes in thick gold films covered with highly optically nonlinear polydiacetylene layers have been studied. Measurements of photon tunneling through individual nanometer scale pinholes has provided strong indication of "photon blockade" effect similar to Coulomb blockade phenomena observed in single-electron tunneling experiments [1]. We have also reported observation of photon tunneling being gated by light at a different wavelength in similar but somewhat larger pinholes [2]. These observations suggest possibility of building a new class of "gated" photon tunneling devices for all-optical signal and image processing in classical and quantum communications and computing. A big step forward would be to artificially fabricate such devices in controllable and reproducible manner. Moreover, massive parallel all-optical signal processing can be achieved if large arrays of such pinholes with enhanced nonlinear properties can be created. However, transition from individual pinholes to a periodic array of them leads to new physics involved in the nonlinear optical processes.

Linear optical properties of metallic films perforated with an array of periodic subwavelength holes and exhibiting the so-called extraordinary enhanced optical transmission have been intensively studied recently (see [3] 5] and Refs. therein). Although the exact nature of the enhanced transmission in two-dimensional case (which is significantly different from a better understood one-dimensional case of subwavelength slits) is still not fully established, the role of surface plasmon polaritons (SPPs) in this process is commonly accepted. SPP Bloch waves can be excited by incident light on the interfaces of a periodically nanostructured film, which may be treated as a surface polaritonic crystal [5] [7. Resonant light tunneling via states of surface polariton Bloch waves is one of the mechanisms involved in the enhanced transmission.

A fundamental difference between conventional photonic crystals and surface polaritonic crystals is a different electromagnetic field distribution close to the surface. Surface polariton is an intrinsically two-dimensional excitation whose electromagnetic field is concentrated at a metal interface. Thus, in contrast to photonic crystals, strong electromagnetic field enhancement takes place at a SPP crystal interface related to the surface polariton field localization [8]. Such enhancement effects are absent in photonic crystals. Field enhancement results in much stronger nonlinear response achieved with surface polaritonic crystals as it is related to the local field strength. This can significantly facilitate nonlinear optical mixing in such structures.

As a logical step forward, we can ask what would happen if a periodically nanostructured metal film would be embedded in a nonlinear optical material. In this Letter we report first experimental studies of nonlinear optical properties of arrays of artificial nanometer-scale cylindrical pinholes in free standing thick gold films, which exhibit strong nonlinear optical mixing due to the presence of nonlinear material inside the pinholes and on the interfaces of the film. In addition to the SPP effects on the film interfaces, due to pronounced cylindricality of the holes, the shape-resonances (cylindrical surface plasmons) can be excited in the holes [9]. At a given transmitted optical power, for a small cylinder diameters optical field of a cylindrical surface plasmon grows inversely proportional to the diameter. Thus, sufficiently thin cylindrical pinholes filled with nonlinear optical material should start to exhibit enhanced nonlinear optical mixing.

In our experiments rectangular arrays of nanometric cylindrical holes have been patterned into a freestanding $400 \mathrm{~nm}$ thick gold membrane using focused gallium ion beam (FIB) milling. To avoid stress in the gold membrane, the gold film is supported by $50 \mathrm{~nm}$ thick $S i_{3} N_{4}$ membrane with $5 \mathrm{~nm}$ intermediate $\mathrm{Cr}$ layer. Since there is no glass/metal interface, milling artifacts such as redeposited material can be avoided leading to a betterdefined structure geometry. We have been successful in patterning relatively large areas $(500 \mu \mathrm{m} \times 500 \mu \mathrm{m})$ of the gold membranes, creating nanosized holes with dimensions down to $20 \mathrm{~nm}$. The diameter of the hole can be controlled by using a controlled endpoint termination technique. In this technique a Faraday cup is placed underneath the membrane and the transmitted ion current is measured in-situ during FIB milling. The milling can then be terminated, by blanking the ion beam, once a 
defined ion current is passing through the emerging hole in the membrane. The images of the cylindrical pinhole arrays fabricated in a $400 \mathrm{~nm}$ thick free standing gold film are shown in Fig. 1. Similar to the sample preparation described in [1], a drop of poly-3-butoxy-carbonylmethyl-urethane (3BCMU) polydiacetylene solution in chloroform was deposited onto the gold film surface. After solvent evaporation films of polydiacetylene were left on both the top and the bottom surfaces of the perforated gold film. Such 3BCMU polydiacetylene films hold the current record for the largest fast nonresonant optical $\chi^{(3)}$ nonlinearity [10].

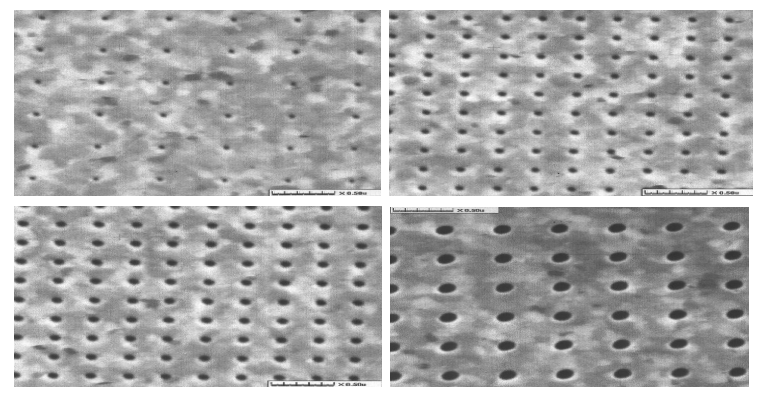

FIG. 1. Focused ion beam milled arrays of cylindrical pinholes in a $400 \mathrm{~nm}$ thick free standing gold film. The scale bar is $500 \mathrm{~nm}$.

Our experimental setup is shown in Fig.2. A bent optical fiber tip similar to the bent tips often used in near-field optical microscopes [11 was used to collect the transmitted light. The fiber tip was positioned above the individual pinhole array in contact with the thick polymer coating using a far-field optical microscope and the shear-force distance control system commonly used in near-field optical setups. [12] Since pinhole arrays were typically separated by at least $10 \mu \mathrm{m}$ distances, properties of individual arrays were studied in the absence of any background scattered light. Under far-field microscope control, $\sim 30 \times 30 \mu \mathrm{m}^{2}$ area of the sample containing the pinhole array of interest has been illuminated with $488 \mathrm{~nm}$ light of a CW argon ion laser and the $633 \mathrm{~nm}$ light of a CW He-Ne laser. The latter was used as signal light and the former was used as control light in the optical gating experiments. The samples were illuminated at the angle of incidence of approximately $45^{\circ}$ so that the projection of the illuminating light wavevector onto the surface is in the direction approximately corresponding to the direction along the hole rows $(\Gamma-X$ direction of the Brillouin zone).

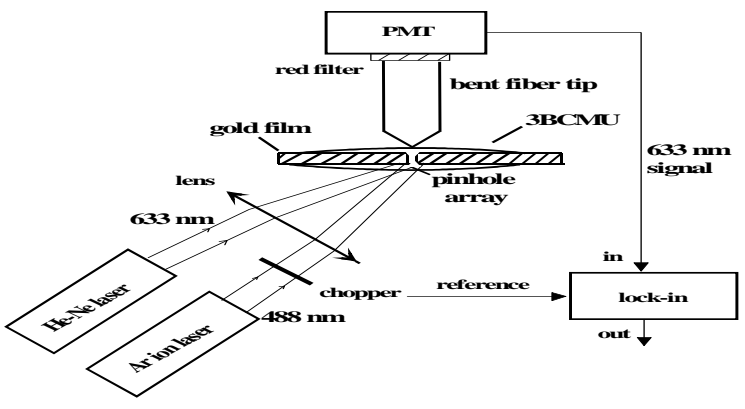

FIG. 2. Schematic view of the experimental setup.

First we have studied how transmission of the arrays at the signal light wavelength $(\lambda=633 \mathrm{~nm})$ is affected by simultaneous illumination with control light $(\lambda=488 \mathrm{~nm})$. In order to observe light controlled tunneling directly, we modulated the incident $488 \mathrm{~nm}$ light with a chopper at a frequency of $1.2 \mathrm{kHz}$. Light transmitted through the pinhole array was collected with the fiber and detected with a photomultiplier (PMT) through an optical filter which completely cuts off the blue light. Modulation of the transmission of the array with $20 \mathrm{~nm}$ holes at the $633 \mathrm{~nm}$ wavelength induced by modulation of P-polarized control light illuminating the same area was observed using a lock-in amplifier, which was synchronized with the chopper (Fig. 2). Thus, the intensity variations of the signal light tunneling through the pinhole array induced by $488 \mathrm{~nm}$ light have been measured directly (Fig. 3). The time behavior of the observed switching is determined by the time constant of the lock-in-amplifier $(\sim 3$ s) needed for the signal integration. At the same time, for the arrays with larger holes $(\sim 100 \mathrm{~nm}$ diameter $)$ no significant variations of the transmission was observed for the given wavelengths and intensities of signal and control light.

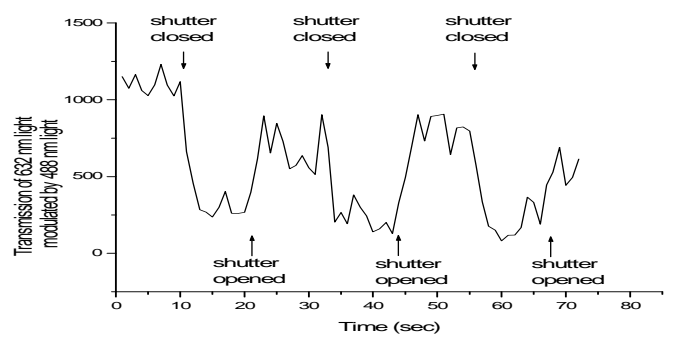


FIG. 3. Modulation of the $20 \mathrm{~nm}$ pinholes array transmission at $633 \mathrm{~nm}$ induced by modulation of control light. The shutter of control light was closed and opened three times during the experiment.

Polarization properties of the signal light passing through the array of smallest pinholes are strikingly different from the polarization properties of the transmitted red light measured in the absence of blue light illumination. These polarization properties provide a very strong proof of nonlinear optical mixing in the polydiacetylenefilled pinholes. In the absence of modulating blue light, transmission of P-polarized red light is higher than the transmission of S-polarized red light by approximately a factor of 3 . This observation is consistent with the theoretical model of the enhanced transmission of subwavelength pinhole arrays relying on the excitation of surface polaritons on both film interfaces, since at the oblique incidence the light of different polarizations interacts with different SPP resonances. This ratio of Pto S-polarized light transmission has been observed to be rather insensitive to the pinhole diameter in a studied range of the pinhole sizes as is expected since the spectrum of the SPP excitations depends mainly on the periodicity of the structure.

However, under the modulation with P-polarized blue light, the $\mathrm{P}$ to $\mathrm{S}$ ratio in the modulated red light transmission jumps to approximately a factor of 20. Polarization measurements offer the best distinction between nonlinear optical mixing effects of interest and possible thermal effects such as thermal expansion of the pinholes, heating of the filling material etc. which could interfere with the nonlinear optical effects. Thermal modulation of subwavelength aperture transmission has been observed previously under intense aperture illumination [13. We were able to observe similar effects in the pinhole arrays transmission at much larger than usual illumination intensities achieved by much tighter focusing (to within a-fewmicrometers spots) of the modulating blue light. However, polarization dependence of the modulated red light obtained under such conditions is very weak (Fig.4(c)): the $\mathrm{P}$ to $\mathrm{S}$ ratio in the thermally modulated red light transmission falls to approximately a factor of 1.5.

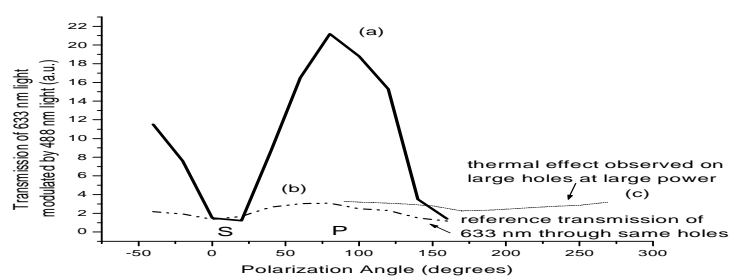

FIG. 4. (a) Polarization dependence of the modulation of the $20 \mathrm{~nm}$ pinholes array transmission at $633 \mathrm{~nm}$ induced by modulation of P-polarized $488 \mathrm{~nm}$ light. (b) Polarization dependence of the same array transmission at $633 \mathrm{~nm}$ without illumination with blue light. (c) Polarization dependence of the thermal modulation of the red light transmission.

Extremely strong polarization dependence of the modulated transmission of the signal light can be explained taking into account the properties of surface polariton Bloch waves on the periodically perforated gold surfaces, the properties of surface plasmons in cylindrical channels, and the properties of a $\chi^{(3)}$ nonlinear susceptibility tensor of 3BCMU polydiacetylene.

To understand the observed transmission behavior, let us consider the spectrum of electromagnetic excitations in our system. At certain combinations of incidence angles and wavelengths, the electromagnetic wave incident on the periodic structure excites the surface polariton Bloch waves. Diffraction of light on a periodic structure provides the wave vector conservation needed for the coupling to SPP [8]:

$$
\mathbf{k}_{S P}=\frac{\omega}{c} \sin \theta \mathbf{u}_{x y} \delta_{s-p} \pm p \frac{2 \pi}{D} \mathbf{u}_{x} \pm q \frac{2 \pi}{D} \mathbf{u}_{y}
$$

where $\delta_{s-p}=1$ for $\mathrm{P}$-polarized incident light (defined with respect to the film interface) and 0 for S-polarized light, $\mathbf{k}_{\mathbf{S P}}$ is the surface polariton wave vector on a periodic interface, $\mathbf{u}_{x y}$ is the unit vector in the direction of the in-plane component of the incident light wave vector, $\mathbf{u}_{x}$ and $\mathbf{u}_{y}$ are the unit reciprocal lattice vectors of a periodic structure, $\mathrm{D}$ is its periodicity (assumed to be the same in both $\mathrm{x}$ - and $\mathrm{y}$-directions), and $\mathrm{p}$ and $\mathrm{q}$ are the integer numbers corresponding to the different propagation directions of the excited SPP modes. Since SPP is in general a longitudinal excitation, the electric field of the excitation light should have an electric field component perpendicular to the surface or parallel to the SPP propagation direction. Therefore, according to Eq.(11) surface polaritons excited with S-polarised light correspond to the Bloch waves at the edge of the even Brillouin zones (standing modes), while for P-polarized excitation they are propagating Bloch waves. 
In our case, the metal film is sufficiently thick to neglect the interaction between surface polaritons excited on different interfaces, therefore two independent sets of resonances can be considered 80. Since one side of the metal film is covered with a polymer $(\epsilon \approx$ 1.7) while another is in contact with $\mathrm{Cr} / \mathrm{Si}_{3} \mathrm{~N}_{4}(\epsilon \approx$ 2.0 ), the SPP resonant conditions will be slightly different on different interfaces of the film. (The dispersion of surface polaritons on a smooth surface is given by $k_{S P}=\omega / c\left(\epsilon \epsilon_{m} /\left(\epsilon+\epsilon_{m}\right)\right)^{1 / 2}$, where $\epsilon_{m}$ and $\epsilon$ are the dielectric functions of metal and adjacent medium, respectively.) Since the filling factor of the structures under consideration is small enough $\left(f=(d / D)^{2}\right)$ the modifications of the SPP dispersion on a smooth interface is expected to be also small, and it can be used to estimate the resonant frequencies of the SPP Bloch waves excitation. Considering $\Gamma-X$ direction of the Brillouin zone $(p= \pm 1, \pm 2, \ldots ; q=0)$, for $\mathrm{P}$-polarised incident light and the angle of incidence of $45^{\circ}$ used in the experiment, the SPP Bloch waves corresponding to 3rd and 4th Brillouin zone are excited at the wavelengths close to $\lambda=633 \mathrm{~nm}$ on the polymer-metal interface. The SPP states related to the illuminated $\left(\mathrm{Si}_{3} \mathrm{~N}_{4}\right)$ interface will be red-shifted with respect to this frequency. At the same time for S-polarised light, the resonant SPP frequencies on the metal-polymer interface are far away from the signal light frequency while SPP Bloch waves from the 4th Brillouin zone of the illuminated interface are relatively close to it. This SPP is about twice weaker than the SPP on a pure gold surface due to presence of a $\mathrm{Cr}$ layer which has strong losses at this wavelength $(\operatorname{Im\epsilon } \approx 30)$. Analogous picture can be constructed in the $\Gamma-M$ direction of the SPP Brillouin zone, but the related resonant frequencies $(p, q)=( \pm 1, \pm 1)$ will lie between the frequencies determined by $(p, q)=( \pm 1,0)$ and $(p, q)=( \pm 2,0)$, and therefore, far from the signal wavelength. However, these resonances can become important for other angles of incidence.

To complete the picture, the resonances associated with cylindrical channels in a metal film should be considered [9]. For thick films with well defined cylindricality of the channels $(d<<h)$, the spectrum of surface electromagnetic excitations in channels has a rather complicated structure with both radiative and nonradiative modes present. Individual cylindrical channels will have a discrete spectrum of resonances asymptotically approaching surface plasmon frequency $\left(\epsilon_{m}=-\epsilon\right)$ from the high frequency side. For polymer-filled channels these modes overlap the frequency of the control blue light for large quantum numbers $s>>1$. The interaction between channels in an array can additionally broaden these resonances leading to minibands [14]. Thus, quasi-continuous spectrum of the states related to the cylindrical surface plasmons can be expected in the spectral range of the control light. For infinitely long cylindrical channels the spectrum of wave vectors along the cylinder axis $h_{z}$ is continuous. Physically one can imagine these excitations as surface modes of a spiral trajectory on a cylinder chan- nel surface. Real (nonradiative) surface modes can not be excited directly by light, but at the frequency corresponding to the control light the very long wave vector SPP can be excited on the periodically perforated polymer-metal interface, which then can be coupled to cylindrical surface plasmons.

In the absence of control light, the transmission of red light takes place via resonant tunneling through the states of SPP Bloch waves on the polymer-metal interface. The changes of the incident light polarization result in the shift of the SPP resonances and, hence, variation of the transmission. Being confined to the polymer-coated interface, the SPP modes responsible for this transmission are very sensitive to the dielectric constant of the polymer, since changes of the dielectric constant modify the SPP resonant conditions and the transmission coefficient. Control (blue) light coupled into cylindrical surface plasmons either via their radiative part or via surface polaritons results in the local changes of the dielectric constant of the polymer due to third-order Kerr nonlinearity. Local electromagnetic field is strongly enhanced in and around the channel due to cylindrical surface plasmons excitation because of small volume of these surface modes $\left(E_{L} \sim 1 / d\right)$.

The polarization properties of the polymer molecules themselves may play significant role in the observed increase of the $\mathrm{P}$ to $\mathrm{S}$ ratio in the modulated transmission of red light. The third-order nonlinearity of 3BCMU polydiacetylene is contributed mainly by the $\pi$-electrons in the backbone of the polymer [10]. Each straight segment of polymer backbone could be treated as identical onedimensional rod-like chromophore. At the microscopic level the $\chi^{(3)}$ tensor of 3BCMU is dominated by only one component: $\chi_{\text {ssss }}^{(3)}$, where $s$ is the direction of the polymer chain. This fact has been verified in measurements of different components of the macroscopic $\chi^{(3)}$ tensor of spin-coated thin polymer 3BCMU films, where polymer backbones have random in-plane orientations, determined by a flat substrate. 10. In our case, the preferential direction for the long polymer backbones inside narrow cylindrical channels ought to be the direction along the channel. The nonlinear optical mixing of interest is determined by the components $D_{l}\left(\omega_{2}\right)$ of the optical field inside the channels at $\lambda_{2}=633 \mathrm{~nm}$ :

$$
D_{l}\left(\omega_{2}\right)=\chi_{i j k l}^{(3)} E_{i}\left(\omega_{1}\right) E_{j}\left(\omega_{1}\right) E_{k}\left(\omega_{2}\right),
$$

where $\omega_{1}$ and $\omega_{2}$ are the frequencies of blue and red light, respectively. If the zzzz-components (along the channel direction) of $\chi_{i j k l}^{(3)}$ dominate the third-order susceptibility, the modulated red light must be strongly P-polarized. At the same time, at the frequency of the blue light $\omega_{1}$ the electromagnetic field in the cylindrical channels is dominated by the plasmon modes with large wave vectors along the channel, for which optical field oscillations have significant longitudinal component, which is along the channel direction. Thus, one should expect the enhanced nonlinear optical mixing to occur while electromagnetic 
field is traveling through small cylindrical holes.

In conclusion, we have reported first observation of photon tunneling gated by light at a different wavelength in an artificially created array of nanometer scale cylindrical channels in a thick gold film. Polarization properties of gated light provide strong proof of the enhanced nonlinear optical mixing in nanometric channels involved in the process.

[1] I.I. Smolyaninov et al., Phys. Rev. Lett. 88, 187402 (2002).

[2] I.I. Smolyaninov et al., cond-mat/0205160.

[3] T.W. Ebbesen et al., Nature (London) 391, 667 (1998).

[4] A. Krishnan et al., Optics Comm. 200, 1 (2001).

[5] L. Salomon et al., Phys. Rev. Lett. 86, 1110 (2001).

[6] N. E. Glass and A. A. Maradudin, Phys. Rev. B 29, 1840 (1984).

[7] W. L. Barnes et al., Phys. Rev. B 54, 6227 (1996).

[8] H. Raether, Surface Plasmons, Springer, Berlin, 1988.

[9] Electromagnetic Surface Modes, A.D. Boardman, Ed., John Willey, New York, 1982.

[10] K. Yang et al., Optics Comm. 164, 203 (1999).

[11] A. Lewis et al., Ultramicroscopy, 61, 215 (1995).

[12] E. Betzig et al., Appl. Phys. Lett. 60, 2484 (1992).

[13] D.I. Kavaldjiev et al., Appl. Phys. Lett. 67, 2771 (1995).

[14] V. Kuzmiak et al., Phys. Rev. B 50, 16835 (1994). 